\title{
Laboratory turnround time: closing the loop
}

\author{
W S A Smellie, P J Galloway, J I Johnston
}

\begin{abstract}
Aims-To institute recommendations from a laboratory turnround time study; to evaluate audit methods; and to quantify improvements achieved.

Methods-Changes to result report distribution and specimen delivery were affected by posting results directly from the laboratory followed by the introduction of a twice daily courier service. Improvements were evaluated by repeating the turnround time audit described in an earlier report. Pre-, peri- and postanalytical turnround times were compared before and after changes had been instituted.
\end{abstract}

Results-Directly posting general practitioner (GP) results increased the percentage of reports which reached their destination within one and two days after they were generated from 13 to $29 \%$ and from 68 to $82 \%$, respectively. Pre- and postanalytical times were superimposable before and after posting was started. Corresponding improvements to the satellite hospital service were from 25 to $78 \%$ and from 60 to $82 \%$, respectively. The courier service shortened the median total turnround time from 50 to nine hours for GPs and from 69 to 18 hours for the satellite hospital. Fifty three per cent of GP reports and $21 \%$ of satellite hospital reports arrived on the same day as the sample was taken: $99 \%$ and $94 \%$, respectively, had arrived by the next day. The number of analytically "old" samples which arrived the day after they had been taken, thus invalidating many results, fell from 25 to $3 \%$. Conclusions-These audits of laboratory turnround time have been used to present a valid case for changes to laboratory transport and to quantify the improvements achieved. They produce consistent and repeatable results, which may also be used to monitor future performance, to assess further changes and to establish the cost-effectiveness of resources used.

(f Clin Pathol 1995;48:372-375)

Keywords: Audit, analytical times, core analyses.

Institute of Biochemistry, Infirmary, Castle Street Glasgow G4 0SF W S A Smellie P J Galloway J I Johnston

Correspondence to: Dr W S A Smellie. Accepted for publication 25 August 1994

The Audit Commission recently recommended changes to laboratory practice to reduce nonanalytical delays in handling samples and results. ${ }^{1}$ The human resource implications of undertaking repeated audits of sample turnround times is, however, a potentially limiting factor in improving laboratory performance. There is sessing laboratory turnround time; firstly, to be able to present a valid case to service users and budget holders for the need for specific targeting of resources and, secondly, to confirm that, once allocated, this results in material improvement to the laboratory service which can be translated into real improvements in patient care. Published turnround time assessments have, to date, centred principally on the analytical phase of sample handling, ${ }^{2-4}$ or where total turnround time has been evaluated, have applied the turnround time model to the stat test, ${ }^{5-8}$ one in which the pre- and postanalytical phases have, at least in principle, been minimised by rapid sample delivery methods and direct telephoning (or other direct transfer) of results to the requesting centre.

We have recently applied a "needle to notes" model of routine laboratory turnround time ${ }^{9}$ to examine sample handling from several laboratory users: three wards in the core hospital, four local general practitioner (GP) centres (within three miles across city traffic) and one satellite psychiatric hospital (two miles across city traffic). We assessed routine main analyser tests over about four weeks and reported on the fate of 436 result reports. We found that in the routine service to all users the postanalytical phase accounted for $64-88 \%$ of total turnround time, the pre-analytical phase for $7-17 \%$, and the analytical phase for $2-29 \%$. The range of postanalytical times was between 18 and 122 hours. Twenty five per cent of the GP samples evaluated arrived the day after being taken and therefore were analytically invalid for most routine measurements. We also found that the length of the analytical period could be highly dependent on sample delivery times, as later deliveries were incompatible with same day analysis.

This report describes changes to the sample delivery and result report distribution services made since the original study, and a repeat audit of pre-, peri- and postanalytical turnround times after these changes had been instituted.

\section{Methods}

Repeat turnround time audits were performed on results sent to the same centres as assessed in the previous study following institution of the changes described below. a need for easily repeatable methods of as-
CHANGES INSTITUTED

Direct posting of results

Based on the results of the initial turnround time study, the laboratory prepared a detailed case for allocation of resources to post results directly and thereby bypass the hospital's in- 
ternal mail system. This case was accepted for a two month trial period, initially to $20 \mathrm{GP}$ and hospital centres. Result envelopes were posted "first class" on a once daily basis at 1730 hours, in a central Post Office close to the hospital, by a member of the laboratory staff.

\section{Courier service}

Although significant improvements were achieved by posting results directly, this change has no effect on pre-analytical or analytical turnround times and limits the optimal postanalytical turnround time to overnight delivery or about 16 hours. Therefore, a case was presented to the hospital for a twice daily courier service, tailored to GPs' needs, both for collection of samples and distribution of result reports. The service was designed to serve user GP centres and local hospitals, to collect samples and distribute results at times calculated to coincide with the end of GP morning and evening surgeries. Tenders were invited and the service commenced in May 1994.

\section{TURNROUND TIME STUDY \\ Postal changes}

The effects of changing the postal system were evaluated by repeating the turnround time study using identical methodology to that described in our earlier report, in the same GP and satellite hospital centres. A trial period of two weeks was chosen to provide about 50 results per centre. Preliminary preparation was minimal; log sheets were sent directly to each centre after agreement to participate had been confirmed by telephone.

Repeatability of the study method was assessed by comparing the distributions of preanalytical and analytical times which should not be influenced by changes in the method used to deliver reports. Postanalytical times before and after introduction of the pilot postal scheme were then compared by examining the numbers of reports which reached their destination within one and two days (time limits of 32 and 56 hours, respectively, from the beginning of one working day until the end of the second or subsequent day).

\section{Changes resulting from the courier service}

Changes after introduction of the courier service were evaluated in the same centres using the same methodology as described previously, with the exception that report arrival times were extrapolated from the despatch times

Table 1 Changes in the percentage of reports which reached their destination within one (32 hours) or two (56 hours) days of report generation following introduction of changes to the postal system

\begin{tabular}{lllll}
\hline Requesting centre & Time & Initial review & Second review & Improvement (\%) \\
\hline GPs & 32 hours & $13 \%$ & $29 \%$ & $120 \%$ \\
Satellite hospital & 56 hours & $68 \%$ & $82 \%$ & $20 \%$ \\
& 32 hours & $25 \%$ & $78 \%$ & $310 \% *$ \\
& 56 hours & $60 \%$ & $82 \%$ & $36 \%$ \\
\hline
\end{tabular}

* This figure, in part, reflects an increase in the number of samples analysed early in the day after they were received as a result of the evening collection. which were recorded by laboratory staff immediately before envelopes were collected by the courier in the laboratory and not by the recipient GP or satellite hospital. We chose to do this to avoid overstretching the goodwill of the centres which had already performed two such studies. As the van arrival times at each centre are detailed accurately on the courier circuit, we were able to time arrival of results as accurately as the recipients.

\section{Results}

POSTAL CHANGES

A total of 167 data points were obtained. These consisted of 117 from the major GP centres and 50 from the satellite hospital. One GP centre was excluded as the number of reports received had increased considerably since the first audit, (33 $v 19 \%$ of all reports) and a much greater proportion of these samples were sent at the end of the working week (33 $v 14 \%$ of reports were posted over a weekend); this would have distorted results and introduced unreasonable positive bias of turnround times.

Pre- and peri-analytical times were plotted separately for samples from GP centres and the satellite hospital. The distribution of preand peri-analytical turnround times for the GP centres was superimposable upon that from the previous study. Samples from the satellite hospital showed an increase in the peri-analytical time which was because of a larger proportion of samples arriving too late in the day for same day analysis.

The percentages of result reports which reached their destination within 32 hours or 56 hours of report generation are shown in table 1. These indicate improvements of between 20 and $300 \%$ in the number of reports reaching their destinations by these times.

\section{COURIER SERVICE}

A comparison of component turnround times before and after the start of the courier service is shown in the figure. These indicate a marked fall in total turnround time for GPs with a median $\mathbf{5 0}$ hours in first review compared with nine hours after institution of the courier service. As a result, $53 \%$ of samples were analysed on a same day basis and a further $46 \%$ were analysed on a next day basis. The major improvement was primarily due to a marked fall in postanalytical turnround time (table 2), although pre-analytical times were also reduced as a result of a twice daily collection tailored to GP surgery requirements. The number of samples which arrived the day after they had been taken also fell dramatically from $25 \%$ before the courier service to $3 \%$ after the service had started. In parallel, the analytical times actually rose (figure) reflecting the number of samples which were received in the evening delivery and which were analysed the following morning. Similar results were found for the satellite hospital ( $94 \%$ cumulative turnround time by the next working day) although the same day turnround time figure $(21 \%)$ was lower, in keeping with the observation that a 

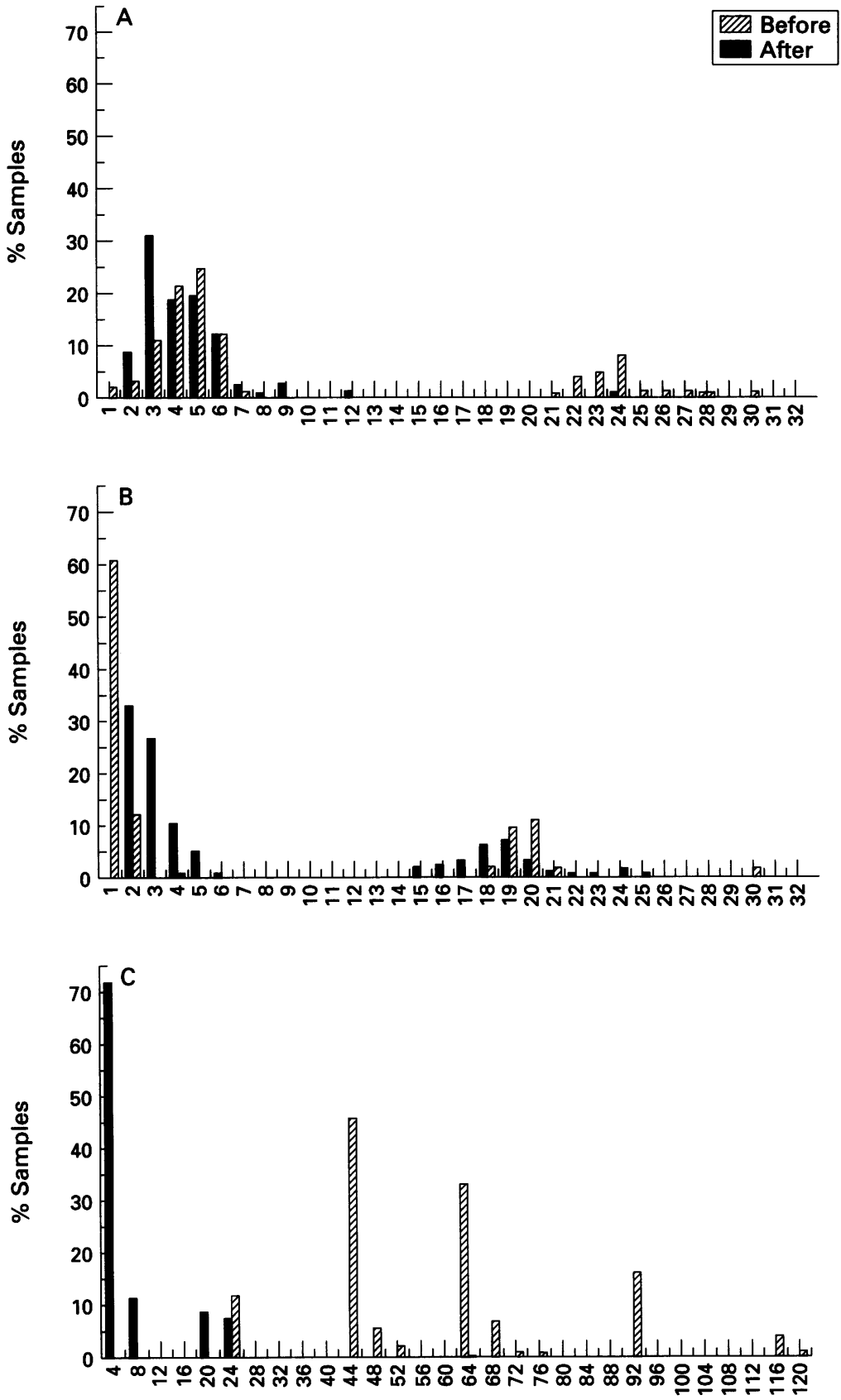

Time (hours)

The distribution of pre-, peri- and postanalytical turnmound times for samples from four GP centres before any changes and after institution of a twice daily courier service. Note that the extreme values over 72 hours are exaggerated in the first audit, as they partly reflect the failure of study centres to record receipt of samples on a Saturday, which is registered in the later method used to evaluate improvements after the introduction of the courier service.

\section{Discussion}

After a preliminary audit designed to identify areas for potential improvement in laboratory turnround time before, during and after analysis, specific changes were targeted at the problems identified in outlying satellite hospitals and the GP centres. These were designed both to improve overall laboratory turnround time, and also to respond to specific needs in terms of the timing of sample collection.

As expected, the postal change produced a clear improvement in postanalytical turnround time and the courier produced changes particularly in postanalytical but also in pre-analytical turnround time.

The postal review demonstrates a clear improvement in the numbers of the results reaching their destination within one or two days of report generation. The figures for GP results are entirely valid as pre- and peri-analytical time distributions were superimposable for the two studies, although those for the satellite hospital should be interpreted with caution as the remarkable improvement, in part, reflects the change in the time when samples arrived in the laboratory.

It is highly unlikely that the figure of about $85 \%$ receipt after two working days could be improved, because, by the methodology used, results posted on a Friday will register a three day (64 to 72 hours) postage time and about $15 \%$ of GP work is posted out over a weekend. Eighty five per cent two working day receipt therefore probably represents an optimal success rate for posting routine work. The most obvious impact of the postal system is the marked improvement in next working day receipt of results.

Although the postal review indicates some scope for improvement within the laboratory in the number of reports which are posted out on the day of analysis, it indicates a viable target time of two days from report generation to destination which could possibly be shortened to one day by further improvements within the department.

Introduction of the twice daily van collection and delivery service had a dramatic effect on both the median overall turnround time and on the percentage of reports being returned to source on a same or next day basis. The two major improvements: faster result delivery and a fall in the number of analytically "old" samples, are likely to have benefitted particularly from the twice daily courier, which is both consistent with same day turnround time and permits late afternoon samples to be collected, reducing the proportion which arrive in the laboratory the day after withdrawal. This also explains the apparent increase in the number of samples with an analytical turnround time in excess of 16 hours, which previously would either simply not have been taken by the GP or would have been received (and analysed) the day after they were taken and would therefore have been analytically invalid for most routine analyses.

The only difference in methodology between the three studies was in the recording of the result arrival time after the courier service had reports were delivered on the day they were generated and $100 \%$ by the following day. The median overall turnround time for the satellite hospital fell from 69 hours in the first review to 18 hours once the courier service had started. 
Table 2 Pre-, peri- and postanalytical turnround times for GP and satellite hospital samples before any changes and after introduction of a twice daily courier service (median values and ranges)

\begin{tabular}{|c|c|c|c|c|}
\hline \multirow[b]{2}{*}{ Site times } & \multicolumn{2}{|c|}{ General practitioner } & \multicolumn{2}{|c|}{ Satellite hospital } \\
\hline & Before courier & After courier & Before courier & After courier \\
\hline $\begin{array}{l}\text { Pre-analytical } \\
\text { no. of samples } \\
\text { median (hours) } \\
\text { range (hours) }\end{array}$ & $\begin{array}{r}150 \\
5 \\
1-71\end{array}$ & $\begin{array}{c}148 \\
4 \\
2-28\end{array}$ & $\begin{array}{c}50 \\
5 \\
3-7\end{array}$ & $\begin{array}{c}33 \\
6 \\
2-23\end{array}$ \\
\hline $\begin{array}{l}\text { Peri-analytical } \\
\text { no. of samples } \\
\text { median (hours) } \\
\text { range (hours) }\end{array}$ & $\begin{array}{r}166 \\
1 \\
1-92\end{array}$ & $\begin{array}{c}197 \\
6 \\
2-30\end{array}$ & $\begin{array}{l}77 \\
20 \\
1-68\end{array}$ & $\begin{array}{c}49 \\
5 \\
2-24\end{array}$ \\
\hline $\begin{array}{l}\text { Postanalytical } \\
\text { no. of samples } \\
\text { median (hours) } \\
\text { range (hours) }\end{array}$ & $\begin{array}{l}166 \\
44 \\
24-120\end{array}$ & $\begin{array}{c}194 \\
2 \\
2-22\end{array}$ & $\begin{array}{l}77 \\
44 \\
16->120\end{array}$ & $\begin{array}{c}49 \\
4 \\
2-21\end{array}$ \\
\hline
\end{tabular}

Note that the extreme values over 72 hours are exaggerated in the first audit, as they partly reflect a failure of study centres to record receipt of sample on a Saturday, which is registered in the later method used to evaluate improvements after the introduction of the courier service.

started. This should not have introduced significant bias with the exception of shortening the recorded delivery time for reports released on a Friday and Saturday which would have been registered as being received on the Monday by the method used in the first two studies. Although this artefactually increases the turnround time for these weekend samples, the overall turnround time for these samples will be recorded as being in excess of 64 hours (Friday afternoon to Monday morning) and therefore represents part of those $15 \%$ of samples at the skewed right tail on the initial graph (figure) and not the main body of samples which have a total turnround time of 24 to 72 hours.

The only significant additional workload introduced by the changes was the need for a member of staff to post result envelopes daily at the end of the day's work, and the need for result reports for the courier service to be available 30 minutes earlier during the working day to enable the courier run to be completed whilst GP surgeries were still open.

We identified in our earlier study that the three different components of turnround time (before, during and after analysis) were interrelated and that changes in one could have knock-on effects on another. It is essential that an audit of turnround times is able to identify these inter-relations and that it can be used to confirm to customers, to the hospital management and to the laboratory alike that improvements have actually been made, and to quantify their effects.

This report has evaluated the reproducibility of a method used to examine turnround times in a biochemistry laboratory and has been used to assess improvements resulting from changes to the laboratory sample and result delivery systems. Its conclusions should be equally applicable to other pathology laboratories, although care must be taken to measure all components of the turnround time cycle as subsequent effects may not be predictable, and practices may change, as seen in the final study in one GP centre and in the satellite hospital following introduction of changes to the postal service. Many improvements may be made at little or no extra cost to the department involved, although others such as a courier service clearly require considerable commitment of resources. As the major user of local delivery services, the laboratories are ideally suited to coordinate such services. This could simplify accountability and facilitate better tailoring of the service to GPs' needs. The resources required can potentially be offset by using such services, once established, to handle the delivery requirements of other parts of the hospital, such as Outpatient mail. This may produce longer term savings by increased costeffectiveness. Improvements can undoubtedly be made, although the issue of final specifications and turnround time "quality" required must remain one between purchaser and provider. It is important that any costs of improvements in service are balanced against actual clinical need, which can only be correctly gauged by active dialogue with the users of the service.

We wish to acknowledge the successful efforts of Drs $F$ Dryburgh and G H Beastall in negotiating and instituting the changes described above, and Miss P Price for her invaluable help in preparing this manuscript.

1 Audit Commission. Working with users. In: Critical Pathology: An analysis of the pathology services. London: HMSO, 1993 9-13.

2 Pellar TG, Ward PJ, Tuckerman JF, Henderson AR. The freckle plot (daily turnround time chart): a technique for timely and effective quality improvement of test turnround times. Clin Chem 1993;39:1054-9.

3 Hilborne LH, Oye RK, McArdle JE, Repinski JA, Rodgerson DO. Use of specimen turnround time as a component of laboratory quality-a comparison of clinician expectations with laboratory performance. Am $₹$ Clin Pathol 1989;92: with lab.

4 Hilborne LH, Oye RK, McArdle JE, Repinski JA, Rodgerson DO. Evaluation of stat and routine turnround times as a component of laboratory quality. Am F Clin Pathol 1989, 91:331-5.

5 Howanitz PJ, Steindel SJ. Intralaboratory performance and laboratorians' expectations for stat turnround times. Arch Pathol Lab Med 1991;115:977-83.

6 Barnett RN, McIver DD, Gorton WL. The medical usefulness of stat tests. Am f Clin Pathol 1978;69:520-4.

7 Howanitz PJ, Steindel SJ, Cembrowski GS, Long TA. Emergency department stat test turnround times. Arch Pathol Lab Med 1992;116:122-8.

8 McConnell TS, Writtenberry-Loy C. Whither waiting: turnround times of laboratory tests of emergency room patients. Lab Med 1983;14:644-7.

9 Smellie WSA, Johnston J, Galloway PJ. A method for assessment of laboratory turnround times: a comparison of Pathol 1994;47:585-8. 